\title{
Creativity in the Age of Robots: A Polemic, Philosophical and Cultural Preface to a Discussion
}

\author{
Gudova Margarita1, Guzikova Maria², Yamshchikov Ivan ${ }^{3}$ \\ ${ }^{1}$ Ural Federal University, Ekaterinburg, Russia \\ ${ }^{2}$ Ural Federal University, Ekaterinburg, Russia \\ ${ }^{3}$ ABBYY, Max Planck Institute for Mathematics in the Sciences, Leipzig, Germany \\ Corresponding author: marggoodova@gmail.com
}

\begin{abstract}
The article mainly argues that education today is the area where "smart machines" such as super-powerful computing technology operate, capable of storing and processing huge amounts of data produced by modern academic researchers, under whose influence new models of human existence - new ontologies - are generated. In addition, there are social "smart machines" for processing information in broadcasting and communication - "new media", which also create their own worlds with their own rules and algorithms; as well as there are personal "smart machines" (gadgets), in which information processing algorithms are reproduced in the form of programs that are created by scientists and programmers, and on the basis of which reading, information analysis and decision-making takes place in the memory of a "smart machine".

Keywords: age of robots, creativity, creative intelligence, human intelligence, artificial intelligence
\end{abstract}

\section{Gudova M.:}

For a long time, man as a species considered himself the only bearer of intelligence on the planet. The intellectual exclusivity of man was presented by many generations of thinkers as a decisive advantage of man over all other living beings and the natural world as a whole. Gradually, in the history of culture, robots became part of human life. They were used as machines and mechanisms capable of acquiring and analysing information and making decisions. Today, artificial systems can have physicality, sensibility and intelligence meaning that they can make decisions. From the point of view of improving the ability of machines to process information, the entire history of human culture is just a preamble to the modern stage, when robots 
became part of our everyday lives, were spread to production and various service industries, act as assistants, substitutes and prostheses of certain organs and human systems like exo- or 3D prostheses. Media are exactly the same 'prostheses'. M. McLuhan said that they are an artificial extension of man; and at the same time, that means the separation and transfer of a part of the human to a machine, e.g. the ability to memorise, transmit and broadcast information. The twentieth century, as defined by M. McLuhan, is the 'age of the redistribution of consciousness', 'Rapidly, we approach the final phase of the extensions of man - the technological simulation of consciousness, when the creative process of knowing will be collectively and corporately extended to the whole of human society, much as we have already extended our senses and our nerves by the various media' [McLuhan, 5]. From the point of view of modern media research, one of the key scientific ideas of M. McLuhan is the description of the social and cultural consequences of media. For M. McLuhan, media were associated with texts and languages. One after one, he describes the means of production, storage and transmission of information, as well as the means of communication that appeared at the turn of the 19th and 20th centuries and appropriated the functions of perception, storage, processing and transmission of information, and how they changed the way of life, the object-material world, habits and forms of communication between people. The media's capability of performing these functions transformed the world during the 20th century in such a way that the cultures of the megalopolises of the late 19th and early 21st centuries differ in a striking way in both technical and technological saturation and in terms of existential issues.

For us, robots in modern education is the relevant target of interest. In our opinion, education today is the area where "smart machines" such as super-powerful computing technology operate, capable of storing and processing huge amounts of data produced by modern academic researchers, under whose influence new models of human existence - new ontologies are generated. In addition, there are social "smart machines" for processing information in broadcasting and communication - "new media", which also create their own worlds with their own rules and algorithms; as well as there are personal "smart machines" (gadgets), in which information processing algorithms are reproduced in the form of programs that are created by scientists and programmers, and on the basis of which reading, information analysis and decision-making takes place in the memory of a "smart machine". 
Why is the education sector so sensitive today to the creation of new ontologies and algorithms? The answer seems to be obvious: from an anthropological point of view, the education system is that of human-to-human communication, 'processing people by people' [Marx, Engels, 29]. When intermediaries in the form of complex intellectual systems and algorithms appear in the relations between people, then a person finds himself/herself in a new existential context, the human world becomes a human-machine world, the ontology of the human turns into an ontology of hybrid existence, and a person feels existential tension in his/her existence between human and inhuman. How do man-made algorithms cause this existential discomfort? Being in a hybrid human-machine world requires considering the peculiarities of not only a person as a partner in educational activities but also an algorithm. In the case of industrial use of robots, humans exploit their ability to perform major repetitive operations with absolute precision an infinite number of times. Industrial robots are slaves to humans, and they are valued for their efficiency; they are ideal performers of heavy and monotonous production operations, freeing people and increasing labour productivity. Service robots in education are another thing. The peculiarity of interaction with 'smart machines' in the education system is that they are not only tools for implementing it but also tools for organising it, algorithms that determine the procedure for and speed of interaction of one actor in the educational process with a machine and then another actor... The set of features associated with the use and organisation allows us to talk about the agent-based active nature of artificial intelligent systems in education, where they act as agents of rational action, by invading the world of human irrationality, impulsivity, emotionality etc. According to B. Cope and M. Kalantzis, the advantage of educational robots is not that they are smarter but that they act logically and consistently, that is reasonably and efficiently.

Let us consider in more detail what kind of artificial intelligent systems an ordinary teacher of a Russian university is dealing with today. This is an incentive scheme for academic staff that collects, places and systematizes information about teachers' work during the academic year, keeps track of their educational, methodological and research achievements. Another agent is a scoring system that collects and posts information about the students' educational problems and the quality of problem solving. It analyses and processes that information, gives advice to users, 
and makes decisions about how successfully the students cope with their tasks based on those points that the teacher gave to the students, and notifies students and teachers about decisions made, either passed or failed. A more complex service robot of the first generation is the Ural Federal University Electronic Open Resources system that not only hosts various electronic courses in its space but also allows limited interactivity with users by posting answers in the form of scanned files or the option of passing tests in an AutoTest mode.

This is intended to provide a composite rating for all these intelligent systems in the methodology set by M. McLuhan in terms of amputations and increments [McLuhan, 5], or, as G. Kress wrote, 'gains and losses' [Kress, 5], or, according to B. Cope and M. Kalantzis, from the point of view of the advantages and disadvantages of artificial intelligence [Cope, Kalantzis, 2019]. First generation educational intelligent systems do not provide any possibility of interactivity. It is possible for such educational robots to use multimodal texts, create archives and interact to a limited extent. The impact that first generation educational robots have on people are tools: they limit and reduce the emotional and impulsive reactions of people in educational interaction and expand the formal and logical abilities, qualities and properties, ensure the sequence of actions and mathematical accuracy of assessments while maintaining out-of-emotionality and value-based neutrality. That is, the first-generation educational robots act as tools for formalising and streamlining the educational relations. That is why the first-generation educational robots, intelligent systems, that do not allow full-fledged interaction, have limited algorithms for variable action and are not perfect in design are target for criticism from the point of new literacy pedagogy and new creativity ideas.

Educational robots of the first generation are those algorithms and tools for ensuring and organising the educational process that form a new hybrid ontology of education and today determine the ways of existence of teachers and students, pupils outside any feedback, the empathy and interactivity sought by man. Critical pedagogy proceeds from the premise that 'ontologies should determine algorithms, and not vice versa' [Cope, Kalantzis, Searsmith, 2020, 4]. 


\section{Guzikova M.:}

The coexistence of humans and robots, their competitive relations in those areas, where machines are stronger, more accurate and more dispassionate, more reliable, raise the question of what the new pedagogy should be, and this meets our time's challenges. According to K. Schwab, our new modernity already exists, and it is a digital world in which a person can be replaced by a machine. What will come of this for pedagogy? In the new ontology of the electronic educational environment, which is a law unto itself, the practices that have been developed in traditional education, those instructional concepts and principles that correspond to another ontology - lesson-class, didactic-synoptic - should not be reproduced. Didactic teaching was built on the transfer of knowledge from one teacher to many students, a teacher would have unique knowledge and pass it on 'from mouth to mouth'. As Cope and Kalantzis write, 'artificial intelligence promises a new way forward for assessment and education' [Cope, Kalantzis, Searsmith 14]. Today, the educational situation is fundamentally different, the ways of gaining knowledge and literacy are diverse and they are not limited to the interaction between holders and adepts of knowledge within the scope of formal educational institutions and outside; and in combination with both formal and informal education, modern pedagogical science calls this phenomenon 'individual educational trajectories'. Another aspect of the new literacy is that, in the didactic model of education, the knowledge transfer process was in line with the teacher's ideas about what students should know. Today, the situation has fundamentally changed. The same way as a new consumer-oriented 'on-demand economy' is created, knowledge is also transmitted upon request from a student or his/her representatives planning his/her successful life trajectory. The acquisition of knowledge on social demand of students, the increased search by the consumer in the international open Internet of in search of the necessary professional educational programmes and curricula, makes teachers face competition, meet such requests and offer the most popular and attractive curricula that meet the consumers' and programmes' challenges. This aggravates the issue of the methodological packaging for the knowledge transfer and the content of that packaging, which retains intrigue and interest, generates creative tasks that require finding solutions, as well as the manifestation of student creativity abilities. Therefore, all these reasons make it necessary to look at the educational process from the point of view of pedagogical design, innovative 
methodological support and the most attractive structuring of the content of knowledge. Deep online in the 2 nd generation educational systems enables the teacher to be a designer and co-creator of the educational environment and implement collaborative forms of education. If a machine can not only count and recognise correct answers in closed tests, not only collect but also subject texts to the contextual, semantic and discursive analysis, interpret, unite students and teachers into separate groups on social networks, generate new friendly ontologies, and implement algorithms, which satisfy a person's need for communication in order to support and develop his/her humanity and sociality, allows assessing the students' progress by not only any figures from the teacher but also the opinion of other students and teachers, then the machine becomes an assistant can radically chang in the educational process in the online mode. This is the position of online optimism.

The advantages of an educational system built at the intersection of the intersection of the artificial intelligence achievements (storage and fast processing of big data arrays), 'new media' - social networks and the mobile Internet are that the educational process can be carried out at any time and place, synchronously and asynchronously, convenient and comfortable for introverts and autists, and people with cognitive and physical disabilities in the form of short lectures, multimodal texts of tasks and answers. All these conditions free a teacher from the need to be a lecturer-preacher; it is suggested that he/she becomes a designer of the knowledge received by his/ her students. To do this, the teacher, in our opinion, needs to answer several fundamental and new questions: How does the proposed knowledge meet the needs of the trainees? How well is the transferred knowledge structured? In what language do students master this knowledge better? In the language of verbal text? Audiobooks? Videos? In the language of touch and tactile sensations or in the language of plasticity and music? In the pedagogical interaction all this leads to the need to take into account not only the initial knowledge request for knowledge of students but also their cognitive request for the presentation of material in a certain sign-oriented and semiotic package. A hybrid environment, in which we work together with a machine, the synergy of man and machine, the machine's ability to recognise a text of various nature - verbal, acoustic, visual and/or tactile ensures that the educational process is multimodal and multilingual. The transition from didactic pedagogy to collaborative pedagogy, in which the teacher is the educational process designer, due thanks artificial intelligence, will 
free the teacher from the routine of endless control tests and works, as well as home assignments, as it can be done by a machine, and will give scope for the creative activities by the teacher-designer in not only of educational programmes, courses and lessons but also social groups, and social and human relations in the educational process by trying out the best human qualities, such as kindness, responsibility, honesty and creativity.

\section{Yamshchikov I.:}

Is it difficult to train a machine to be a teacher's assistant, solve creative problems and evaluate the quality of the obtained solutions? Is it difficult to teach artificial intelligence to be creative and generate texts that are read like human-written texts? What are the creative possibilities of artificial intelligence and where are its limits? Can a modern computer surpass human intelligence in its creative capabilities?

On the one hand, Turing writes in his article on statistical learning [1]: "If the meaning of the words "machine" and "think" are to be found by examining how they are commonly used it is difficult to escape the conclusion that the meaning and the answer to the question, "Can machines think?" is to be sought in a statistical survey such as a Gallup poll." This starting argument turned out to be prophetic. It pinpoints the profound challenge for the generative models that use statistical learning principles. Indeed, if creativity is something on the fringe, on the tails of the distribution of outcomes, then it is hard to expect a model that is fitted on the center of distribution to behave in a way that could be subjectively perceived as a creative one. If we correlate this idea with the basic principles of statistical learning and try to answer the question about the possibility of creating something new by a machine, then we inevitably come to a disappointing conclusion: the result of a poll will never be something new. In this sense, the machine is able to do what the person taught it. Without creating anything fundamentally new, it re-assembles repeating patterns.

On the other hand, people also reproduce something that they learned from other human beings. In this sense, it is rather difficult to distinguish the happy accident of a serendipitous machine creativity from the accidental insight of an artist. In 2016, Alexey Tikhonov and I conducted a series of experiments: we tried to train an algorithm to generate poems stylized as poems of a given author. The generated poems that resembled the lyrics of the cult Russian poet and musician Yegor Letov became the most widely 
known in Russia. Globally our project with Kurt Cobain stylized songs got more public interest. We recorded two mini-albums (in Russian and in English) with poems generated by the algorithms. The Russian album is called "Neural Defense", and the English one is called Neurona (https:// youtu.be/c759T8zOe5A)*. Both albums are available to listen to and have been published on various streaming platforms. The essence of the project was that we took all available poetic texts in Russian with information about their authorship, and trained the algorithm to create stylized texts similar to the poems of a particular poet. You can read more about this in our articles $[2,3,4]$. Having mastered the basic stylistic techniques of the chosen author, the machine can endlessly produce poems from his vocabulary, based on his rhythmic-melodic techniques. Some of these texts will be better, others worse, but the algorithm itself will not invent any new artistic poetic techniques, it simply uses statistical information that it can extract from the texts in the training set. People reading these poems distinguish well between authors and their style, sometimes machine stylizations are recognized as more 'human' than the original poems. Generally, the readers have hard times distinguishing the author and the machine. Modern generative algorithms tend to pass Turing test in terms of stylization. In some sense, artificial intelligence creates the best postmodern works today. The contribution of algorithms to culture is the death of postmodernism.

There are experiments in other creative fields where algorithms generate music and graphics using statistical learning. But to which extent are these pieces novel? In order to understand this, one can regard the limits of creative artificial intelligence within a broader problematic field of communication. We interact with machines, but we do not communicate with them. There are two phases in machine learning process: exploration and exploitation. A person goes through the same phases both in phylogeny and ontogenesis. In humans, over the years, the research phase is increasingly displaced by the exploitation phase. A person develops certain algorithms - habitual, ritual actions, - and begins to reproduce them in her life. When we are kids, the task "go there - don't know where, bring that - don't know what" seems exciting and entertaining. Over the years we value clearly defined goals and objectives more and more. The desire for novelty is supplanted

${ }^{*}$ Neurona - In the back of your glass. The lyrics of this song were written by an artificial neural network trained to resemble Kurt Cobain. Neurona is a project of Aleksey Tikhonov and Ivan Yamshchikov. 
by the fear of uncertainty. Thus, the possibilities for creativity are narrowed in the exploitation phase by design. If we expect something new from a machine, then interaction with it should be built on the principle of exploration, rather than exploitation. Meanwhile, the expectation of a predictable outcome is a standard form of our interaction with algorithms. It, by definition, prevents a machine from creating something that a person would perceive as new. We have discussed this way of interacting with algorithms in detail [5]. For example, an interactive stand that allows one to explore the generative capabilities of an algorithm, perceiving it as a certain 'Ding an sich', [6] may be a good example of an installation that facilitates such research interaction between a human and a machine.

Interacting with the algorithm in such an "open" format, you can read the results of their work in a fundamentally different way. In [7], we showed how such an "open" perception can be combined with the creation of several autonomous systems, each of which is responsible for different aspects of the creative process. In the Paranoid Transformer project, a text generation system is combined with a critic system

Interacting with the algorithm in such an "open" format, you can read the results of their work in a fundamentally different way. In [7], we showed how such an "open" perception can be combined with the creation of several autonomous systems, each of which is responsible for different aspects of the creative process. In the Paranoid Transformer project, a text generation system is combined with a critic system, a handwriting generation system, and a system for generating pictures in the margins of a draft. Each of the systems relies on statistical learning algorithms. The final product of their work is the diary of a "mad" digital writer, which is published in hardcover. Here we are no longer talking about stylization. This is a diary of a completely autonomous "author" where everything (from the text itself to the sketches on the margins of a page) is generated algorithmically.

When we say that we would like artificial intelligence to be a human assistant, we should understand that only two alternative modes are realized in interaction with algorithms so far: this are either exploitative interaction or explorative immersion. Interesting is not functional, functional is not interesting. A machine can be a communication partner, and it can be an interesting one, but its functionality will inevitably fade... After all, creation of non-functional, interesting objects is the essence of art itself, be it the Bronze Horseman in St. Petersburg or the graffiti on the First Five- 
Year Plan Square in Ekaterinburg. The creation of non-functional objects that carry the personalized experience of the creator stays one of the main forms of immortality available to us.

\section{Conflict of Interest}

The authors have no conflict of interest to declare.

\section{Acknowledgment(s)}

This research was financially supported by the Russian Foundation for Basic Research (Grant No. 17-29-09136\20 "Multilingualism in the Era of Post-literacy: Philosophical and Cultural Studies and Methodological and Pedagogical Development of a Multilingual Education Model").

\section{References:}

1. M. McLuhan. Understanding Media: The Extensions of Man. Routledge \& Kegan Paul, London (1964).

2. B. Cope, M. Kalantzis, D. Searsmith. Artificial intelligence for education: Knowledge and its assessment in AI-enabled learning ecologies, Educational Philosophy and Theory (2020), 10.1080/00131857.2020.1728732

3. B. Cope, M. Kalantzis. New Media Affordances: Pedagogical Alternatives in Technology-Mediated Learning Environments (2019).

4. K. Marx, F. Engels. Nemetskaya ideologiya. Izbr. proizv. V 3 t. [German Ideology. Selected compositions in 3 volumes]. Moscow, Vol. 1 (1980).

5. K. Schwab. Shaping the Fourth Industrial Revolution. Penguin Random House (2017).

6. G. Kress. Gains and losses: New forms of text, knowledge and Learning. Computers and Composition, V. 22 (2005), pp. 5-22, 10.1016/j.compcom.2004.12.004

7. A. M. Turing. Computing machinery and intelligence. Mind, Vol.59, (1950), pp. 433.

8. I. P. Yamshchikov, A. Tikhonov. Learning literary style end-to-end with artificial neural networks. Advances in science, technology and engineering systems journal, Vol. 4(6), (2019), pp. 115-25.

9. A. Tikhonov, I. P. Yamshchikov. Guess who? Multilingual approach for the automated generation of author-stylized poetry. In Proceedings 2018 IEEE Spoken Language Technology Workshop (SLT), (2018), pp. 787-794.

10. A. Tikhonov, I. Yamshchikov. Sounds Wilde. Phonetically extended embeddings for author-stylized poetry generation. In Proceedings of the Fifteenth 
Workshop on Computational Research in Phonetics, Phonology, and Morphology, (2018), pp. 117-124.

11. I. P. Yamshchikov, A. Tikhonov. I Feel You: What makes algorithmic experience personal? Politics of the Machines-Art and After 7, (2018), pp. 1-6.

12. A. Tikhonov, I. P. Yamshchikov. Drum Beats and Where To Find Them: Sampling Drum Patterns from a Latent Space. In Proceedings of the International Conference on Computational Creativity, Coimbra, Portugal, (2020).

13. Y. Agafonova, A. Tikhonov, I. Yamshchikov. Paranoid Transformer: Reading Narrative of Madness as Computational Approach to Creativity. In Proceedings of the International Conference on Computational Creativity, Coimbra, Portugal, (2020). 